\title{
DENOMINACIONES DE LAS «AVES SILVESTRES» RECOGIDAS DEL ALEANR QUE NO APARECEN EN EL DICCIONARIO DE LA REAL ACADEMIA ESPAÑOLA (1992), $21^{\mathrm{a}} \mathrm{ED}$.
}

\author{
ROSARIO ASENSIO RUIZ \\ (Universidad de Alicante)
}

\begin{abstract}
RESUMEN
This study analyzes the names of wild birds collected in the Aragón, Navarra and La Rioja Linguistic and Ethnographic Atlas which are not recorded in the Diccionario de la Real Academia Española (1992, 21st. edition). The work is organized in five parts. The first part presents the metodology followed in the study and the reasons why the Linguistic Geography must be taken into account when elaborating dictionaries, the second one analyzes the words of the wild birds that should be taken into account by the academic dictionary.

In the third part it appears names that identify different birds to those collected in the DRAE and in the fourth part it is also proved that the words accurate location we are given by the linguistic atlas has not been contrasted by the official dictionary. In the last part the conclusions reached in this study are presented.
\end{abstract}

\section{Introducción}

Entre 1979-1983 salió a la luz el Atlas Lingüístico y Etnográfico de Aragón, Navarra y La Rioja (ALEANR)'. Su autor, Manuel Alvar, justificaba así su aparición:

La realización de un atlas lingüístico y etnográfico de Aragón era una necesidad sentida desde hace años ${ }^{2}$.

Manuel Alvar, con la colaboración de A. Llorente, T. Buesa y E. Alvar, Atlas Linguiistico y Etnográfico de Aragón, Navarra y Rioja, Departamento de Geografia Lingüística, Instituto Fernando el Cátólico de la Excma. Diputación de Zaragoza, CSIC, Zaragoza, 1979-1983, 12 tomos.

2 Manuel Alvar, «Proyecto de un atlas lingüístico de Aragón», recopilado en Estudios de Geografía Lingiaística, Paraninfo, Madrid, 1990, págs. 330-343: pág. 336. 
Cualquier trabajo científico se justifica por sí solo. Pero su importancia y trascendencia dependerá también del tema elegido. La estructura lingüística de Aragón, Navarra y La Rioja presenta una fisonomía bastante compleja. Desde la Edad Media, hemos oído hablar de la «unidad navarro-aragonesa». Esta unión se ha dejado sentir también en La Rioja no sólo en lo geográfico, social y económico sino también en lo lingüístico. El castellano ha influido sobre ella de un modo muy intenso, provocando el empobrecimiento dialectal de estas hablas. Sin embargo, Alvar consideró que era necesario conocer tanto elemento heterogéneo. Así afirma que

El catalán se habla en tierras de Aragón, y en otro tiempo pudo hablarse el vasco. Si no supiéramos otras cosas que esto, merecería la pena intentar conocer tanto elemento heterogéneo. Pero hay más: el Pirineo presenta una serie de hablas, muy erosionadas ya, tan interesantes como las que más lo sean en el noroeste ibérico. Muchos de los rasgos que las caracterizan tuvieron una difusión muy amplia, y la toponimia es hoy el insobornable testigo ${ }^{3}$.

Con la aparición del $A L E A N R$, toda esta heterogeneidad ha quedado bellamente plasmada. Tanta riqueza léxica a la fuerza debía suscitar la atención de los lingüistas. Sabemos que la mayor información que sobre el léxico de una lengua poseemos es la que aparece recogida en los diccionarios. Sin embargo, los repertorios léxicos generales y particulares recogen las voces, en ocasiones, sin contemplar unos criterios determinados, como el uso o la localización geográfica. Gregorio Salvador manifestó a este respecto la importancia de indicar en el diccionario general de la lengua

El ámbito de difusión de cada voz o de cada acepción en los casos en que éstos no sean generales ${ }^{4}$.

Son los atlas lingüísticos los que aportan a los diccionarios la suma del material reunido y cartografiado, proporcionando la distribución geográfica de los términos y, lo que es más importante, dando cuenta de su vitalidad.

Este estudio pretende mostrar, una vez más, la existencia de voces que están suficientemente extendidas por Aragón, Navarra y Rioja y que no constan en el Diccionario de la Real Academia Española. ${ }^{5}$. En este caso vamos a estudiar las denominaciones que reciben las «aves silvestres» en el ALEANR para fijarnos, sobre todo, en aquellas voces que por su extensión y vitalidad deberían figurar en el Diccionario académico.

Loc. cit., pág. 337.

4 Gregorio Salvador, «Voces aragonesas en el DRAE», en Actas del I Congreso de Lingüistas Aragoneses, Departamento de Cultura y Educación, D.L., Zaragoza, 1991, págs. 21-27: pág. 21.

Real Academia Española, Espasa-Calpe, Madrid, 1992, $21^{\text {a }}$ edición. En adelante citaremos por DRAE. 
Un exhaustivo y minucioso examen de cada uno de los mapas referidos a las «aves silvestres» nos ha permitido inventariar y clasificar un total de 1.106 voces que designan a 33 pájaros distintos. La localización exacta de las voces en los mapas lingüísticos ha diferenciado y distinguido el uso y la vitalidad de cada una de ellas. Así, observamos que un total de 571 se registraron, exclusivamente en Aragón, 191 en Navarra y 91 en La Rioja 6 .

Después localizamos las voces en el compendio léxico general de nuestra lengua, el Diccionario de la Real Academia Española. La búsqueda fue desoladora ya que existía, según los mapas lingüísticos, un importante número de voces que estaba suficientemente extendida por Aragón, Navarra y Rioja; sin embargo, muchas de ellas no figuraban en el Diccionario académico. De las 1.106 voces estudiadas el DRAE sólo incluyó en su corpus léxico un total de 199, de las cuales 112 las marcó como generales, 17 como regionales y 70 con un significado distinto al que aparecía en el $A L E A N R$.

No es nuestro propósito entrar en la polémica de cuestionar si el DRAE debe o no incluir dialectalismos. Sabemos que los incluye. Pero queremos objetar que la Academia sigue criterios muy arbitrarios a la hora de introducir en su Diccionario voces dialectales. Tampoco pretendemos que incluya todas y cada una de las voces que hemos registrado en el ALEANR designando a las «aves silvestres». Sería absurdo incluir términos, variantes fonéticas de una voz que hubieran aparecido en una localidad aislada. Ofrecemos sólo aquellos que gozan de una suliciente extensión geográfica — sin embargo, la muestra es bastante amplia-El análisis de los mapas referidos a las «aves silvestres» nos ha permitido comprobar la aparición de voces nunca registradas por el DRAE; nuevas acepciones de las que aparecen en el diccionario académico; localización de voces nunca recogidas o que aunque registradas aparecían en el diccionario sin localización, ya que muchas de las localizaciones del $D R A E$ suelen reducir la extensión real de una voz y, en cambio, muchas de las que se dan como generales debieran limitarse. Nuestro estudio aportará datos que permitirá ampliar, además, determinadas localizaciones o situar las distintas acepciones de una misma palabra, señalando su vitalidad.

Nosotros pretendemos que estas voces tengan un lugar en el diccionario oficial. Los términos que hemos estudiado figuran al menos en tres localidades. Este hecho no es arbitrario ni casual. A este respecto Ana Isabel Navarro afirma que

6 Para poder determinar con exactitud el uso y la localización de un término como propio de Aragón, Navarta o Rioja, realizamos un cotejo de las voces estudiadas con el resto de atlas lingüísticos publicados hasta la fecha elaborados bajo la dirección de Manuel Alvar: con la colaboración de A. Llorente, G. Salvador y J. Mondéjar, Atlas Lingiḯstico y Etnogrúfico de Andalucía (ALEA). Universidad de Granada-CSIC, Granada, 1961-1973, 6 tomos; Atlas Lingüístico y Etnográfico de las Islas Canarias (ALEICan), Ediciones del Excmo. Cabildo Insular, Las Palmas, 1975-1978, 3 tomos; Atlas Lingiaístico y Etnográfico de Cantabria (ALECant), Ed. Arco-Libros, Madrid, 1995, 2 tomos. 
Sabemos que cuando una voz aparece en un punto del mapa salvo error o confusión abarca un área de extensión mucho más amplia que ese lugar determinado. Si figura en tres localidades el área en la que aparece esa voz es considerablemente mayor y digna de ser tenida en cuenta?

\section{Voces}

A continuación, presentamos las voces que deberían ser tomadas en cuenta por la compilación oficial ${ }^{8}$ :

1.- La voz fillesno designa a la 'cría de pájaro' en una localidad de Navarra y en el centro-oeste de Zaragoza". Rafael Andolz definió el término como 'el hijo del animal, polluelo, cachorro' "'. Como 'polluelo, animal de cría' fue registrado por Chusé Araguiés". José María Iribarren recogió la voz como 'cría de pájaro'"12.

2.- La 'pelusa de los pajarillos' recibe la denominación de pelo bruja en el centro-oeste de Navarra ${ }^{13}$. Tomás Buesa explicó que la expresión en pelo bruja se dice del pájaro al que le empieza a salir la pluma ${ }^{14}$.

3.- La locución en pelo bueno designa al 'volantón', pájaro que ha perdido la pelusa que recubre su cuerpo al nacer, ha desarrollado completamente su pelaje y es lo sulicientemente grande para empezar a volar. Esta locución no fue registrada por ningún diccionario a pesar de que fue localizada en gran parte de Rioja, mitad inferior de Navarra y tres localidades en Zaragoza ${ }^{15}$.

7 A. I. Navarro Carrasco, El Atlas de Canarias y el Diccionario Académico, Universidad de Alicante, Alicante, 1996, pág. 22.

8 Respetamos el orden en el que aparecen en los mapas del ALEANR. Este atlas presenta 135 puntos de encuesta, distribuidos de la siguiente manera: 41 en Huesca, 30 en Zaragoza, 36 en Teruel y en Navarra, 21 en Logroño, dos en Vitoria, Guadalajara, Cuenca y Valencia y tres en Castellón. Como en otros atlas lingüísticos, las localidades se presentan por siglas y un número de tres cifras. Las siglas corresponden a las que la provincia tiene en el Ministerio de Obras Públicas. Así, Lo = Logroño, $\mathrm{Na}=$ Navarra, $\mathrm{Z}=$ Zaragoza, $\mathrm{Hu}=$ Huesca, $\mathrm{Te}=$ Teruel, $\mathrm{So}=$ Soria, $\mathrm{Gu}=$ Guadalajara, $\mathrm{Cu}$ = Cuenca $; \mathrm{V}=$ Valencia, y $\mathrm{Cs}=$ Castellón.

Nil 404; Z 300, 303.

10) R. Andolz, Diccionario Aragonés, Mira, Zaragoza, 1992 (4ª edición). En adelante citaremos por Andolz.

11 C. Aragüés, Dizionario Aragonés-Castellán. Castellano-Aragonés, Ligallo de Fablans de l'aragonés, Zaragoza, 1989. En adelante citaremos por Aragüés.

12 J. Ma Iribarren, Vocabulario navarro, Ed. Gómez, Pamplona, 1952. En adelante citaremos por lribarren.

13 Na $301,303,306,307,308,309$.

14 T. Bucsa, Esiudios filológicos aragoneses, Universidad, Zaragoza, 1989, pág. 378. En adelante lo citaremos por Buesa.

15 Bu 400; Vi 300, 600; Lo 100, 101, 102, 300 (en pelo giieno), 302, 303, 304, 305, 500, 501, 502,602 ; Na $304,306,307,308,309,500,501,502,602$; Z 305, 502, 503; So 402. 
4.- Volandero es también 'volantón' en una zona homogénea de Rioja, Zaragoza y Huescal'. En Andalucía se documenta también la voz ${ }^{17}$. El DRAE recogió la forma voladero 'que puede volar', que sólo apareció en una localidad de Teruel $^{18}$.

5.- Chilandre (y vars. fonéts.) identifica al 'gorrión campestre' al oeste de Zaragoza y en localidades aisladas de Teruel ${ }^{19}$. Rafael Andolz define la voz como 'gorrión chillón'.

6.- El 'pinzón' recibe asimismo la denominación de pinchán en algunas localidades de Zaragoza y Huesca ${ }^{20}$. Andolz y Rohlfs ${ }^{21}$ lo relacionan con el «pinzón».

7.- Pinzán designa al 'pinzón' en localidades de Navarra y Huesca ${ }^{22}$. Andolz y Mott $^{23}$ aseguran que identifica al pájaro «pinzón». Rohlfs señala que sólo es un pájaro.

8.- Papirroyo (y var. fonét. papirrojo) es 'petirrojo' en dos puntos de Rioja y en numerosas localidades de Aragón y Navarra ${ }^{24}$. Es el apelativo que dan al pájaro llamado petirrojo, según Iribarren. Aragüés y Rohlfs recogen la variante papirroy como 'petirrojo'. Iribarren dice que la variante piparroyo es 'petirrojo' y que pipirroyo es el 'pájaro que tiene el buche negro'.

9.- Una voz extensamente distribuida por Huesca es cuculo ${ }^{25}$ que designa al 'cuclillo'. Vicente García de Diego ${ }^{26}$ afirma que en latín ya se conocía la forma CUCULUS 'cuclillo'. Es posible que ésta haya permanecido inalterable en aragonés. Andolz y Rohlfs lo definen como 'cuclillo, ave parecida al mochuelo y que se nombra así por el canto'. Andolz recogió igualmente la variante coculo como 'cuclillo'.

\footnotetext{
16 Lo I00, 301, 400, 603; Z 400, 402; Hu 300, 303, 305; V 101.

17 Vid. ALEA, II, adición al mapa 403.

is Te 204.

19) Z 305, 500, 503 (chileandrea), 505, 506 (chilandra), 602 (id.); Te 102, 104 (chilandro), 206, 402 (chilanda), 404 (chilandra); So 402, 600 (chilandro).

20) Z 202; Hu 102, 107, 204, 300.

21 G. Rohlfs, Diccionario dialectal del Pirineo Aragonés, Institución Fernando cl Católico, Zaragoza, 1985. En adclante citaremos por Rohlfs.

$22 \mathrm{Na} \mathrm{502;} \mathrm{Hu} \mathrm{101,207,403.}$

2.3 B. Molt, Diccionario Chistavino-Castellano, Caja de Ahorros de Aragón y Rioja, 1978. En adelante citaremos por Mott.

24 Indicamos con -jo cuando se trata de papirrojo: Vi 600 (-jo); Lo 103 (id.), 502 (id.); Na 104 (id.), 106 (id.), 300, 301 (id.), 302 (; piparroyo), 303 (piparrayo), 304, 307, 308, 309 (pipirroyo), $400(-j o), 40 \mathrm{I}, 402(; i d), 403,404,405,500,501,502,600,603,$.604 ; Hu 100 (paparroy), 101, 102 (papirroy), 104, 106 (papirrey), 107, 109 (papirroy), 110 (id.), 111 (id.), 112 (id.), 203 (id.), 207 (id.), 302 (id.), 406 (pepirroy), 407 (paporroy); Te 200, 203 (-jo), 600 (id.); Cs 300 .

25 Z 202; Hu 101, 102, 105, 107, 108, 203, 204, 206 (coculo), 207 (cubulo), 300, 302 (coculo), 304, 400 (cugulo), 403, 405 (coculo), 407, 500, 600.

25 V. García de Diego, Diccionario etinwológico español e hispánico, Saeta, Madrid, 1969 (2a edición).
} 
10.- El 'cuclillo' también es pecu en un pequeño núcleo de La Rioja ${ }^{27}$. F. González Ollée dice que es un 'ave de plumaje negro, parecido a la paloma', pero no especifica su nombre aunque opina que debe ser el cuco o cuclillo, y añade que en Álava se denomina pecu a esta misma especie. En Cantabria la voz presenta también una clara homogeneidad ${ }^{29}$.

11.- Cucullo es 'cuclillo' en el norte oscense ${ }^{30}$. Andolz la definió como 'cuclillo'. Es curioso advertir que el DRAE sí recogió la voz cuquillo 'cuclillo' la cual apareció también en cuatro localidades aunque en Teruel ${ }^{31}$ ¿por qué no registró de la misma manera cucullo?

12.- Reiseñor es 'ruiseñor' en algunas localidades de Navarra, Zaragoza y Huesca $^{32}$. En asturiano ${ }^{33}$ y leonés ${ }^{34}$ se denomina reiseñor al 'ruiseñor'. Andolz también lo definió como 'ruiseñor'. María Soledad Díez señala que es conocida esta voz es Astorga, Galicia y Argüellos ${ }^{35}$.

13.- El pájaro 'verderón' es verdelón en cinco pueblos riojanos y uno en Vitoria $^{36}$. Gerónimo Borao ${ }^{37}$ registra la palabra con la significación de 'verderol'. Iribarren la documenta en Pamplona y dice que es 'verderón'. Sin embargo, en Navarra no aparece este término. En Andalucía la voz verdelón se halla en cinco localidades almerienses y una granadina ${ }^{38}$. En Cantabria caracteriza dos zonas homogéneas: una al noroeste y otra al sur ${ }^{39}$.

14.- Aloda es 'alondra' en un punto de Rioja, dos de Navarra y de forma general en Huesca ${ }^{41}$. Juan Corominas ${ }^{41}$ afirma que aloda se conserva en el Alto Aragón. Rafael Andolz y Chusé Aragüés lo definen como 'alondra'. Otra expresión que designa a 'alondra' es aloya que se encuentra en el mapa en Rioja

27 Vi $300 ;$ Lo $303,304,305,500,501,502$.

28 F. González Ollé, El habla de la Bureba. Introducción al castellano actual de Burgos, anejo LXXXVIII de la RFE, CSIC, Madrid, 1964.

29) Vid. ALECant, l, adición al mapa 630.

30) Hu 109, 110, 111, 112.

$31 \mathrm{Te} 401,403,404,405$.

$32 \mathrm{Na} 401 ; \mathrm{Z} 200 ; \mathrm{Hu} 110,112,203$.

33 R. d' Andrés, Diccionario Temáticu Asturianu, Alborá Llibros, Asturias, 1991.

34 S. Alonso Garrote, El dialecto vulgar leonés hablado en Maragatería y Tierra de Astorga, Instituto Antonio de Nebrija, CSIC, Madrid, 1947 ( $2^{a}$ edición). En adelante citaremos por Alonso.

$3.5 \mathrm{M}^{2} \mathrm{~S}$. Díez, Léxico leonés, Universidad de León, León, 1994. En adelante citaremos por Dic\%.

30 Vi 600; Lo 101, 102, 103, 302,605.

37 G. Borno, Diccionario de voces aragonesas, Diputación Provincial de Zaragoza, Zaragoza, 1908. En adelante citaremos por Borao.

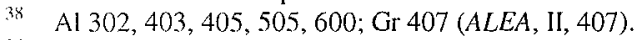

3) $\mathrm{S} 101,201,202,204,205,206,207,208,310,401,500,501,502,600,601$.

4) Lo 602; Na 502, 602; Z 201, 202, 301, 302, 303, 304, 400, 401, 402, 501, 504; Hu 100, $102,103,104,105$ (loda) $, 106,107,108$ (; id) $, 109,110,111,112,206,207,300,301,302,303$, $304,305,405,500$.

41 J. Corominas y J. A. Pascual, Diccionario Crítico Etimológico Castellano e Hispánico, Gredos, Madrid, 1980-1991, 6 tomos. En adelante citaremos por DCECH. 
y Navarra ${ }^{42}$. Esta voz fígura en el DRAE como propia de Álava, Burgos y Rioja con el significado de 'alondra'. Parece ser que la localización en Burgos y Rioja lue posterior ya que Borao al anotar la voz en su Vocabulario dijo que "el DRAE la trae sólo como de Álava,43. González Ollé indica asimismo que aloya es "una voz antigua que el DRAE da como propia de Álava'. Corominas asegura que se utilizaba en Logroño y Burgos. Si observamos en el mapa la distribución de ambas voces, aloya y aloda, advertimos que ésta última se halla en un número mayor de localidades ¿por qué razón no la incorporó también la Academia a su Diccionario?

15.- Recachadera 'alondra' vive en el sudeste zaragozano ${ }^{44}$. Para Andolz es un 'pájaro parecido a la calandria'.

16.- Cucullada (y cocullada) es 'cogujada' en la mitad oriental de Aragón ${ }^{45}$. Andolz y Coll ${ }^{46}$ manifiestan que cucullada es 'cogujada'. El primero registró también la variante cocullada como 'cogullada, cogujada'.

17.- La caliandra 'calandria' aparece en un núcleo homogéneo entre Rioja, Navarra y Aragón ${ }^{47}$. Andolz señala la voz como 'alondra'. Sin embargo, Antoni Alcover $^{48}$ y Benito Coll la definen como 'calandria'. Canarias y Andalucía recogen caliandra, con el sentido de 'alondra'49. En cambio, en Cantabria designó a la "calandria'st.

18.- La 'calandria' es denominada calandra en Huesca y en dos localidades aisladas de Teruel' ${ }^{51}$. Según Andolz y García de Diego identifica a la 'alondra' y para Alonso, Aragüés, Rohlfs y Díez a la 'calandria'. Por otra parte, calandra designa a 'alondra' en Canarias y Andalucía ${ }^{52}$.

19.- El término ocete alude al 'vencejo' en tres localidades de Rioja, una en Zaragoza y otra en Soria'. Cesáreo Goicoechea ${ }^{54}$ afirma que es 'vencejo, pájaro

42 Bu 400; Vi 300, 600; Lo 100, 101 (aloea), 102, 103, 300 (aloya real), 301 (loea), 302 (dloea), 303, 304, 305, 400 (id.), 401, 502, 601 (id.), 603 (id.), 604; Na 306 (id.), 309 (id.).

G. Borao, Vocabulario aragonés, op, cit.

$\mathrm{Z} 600,601,602,603$.

Z 101, 202, 400 (cocullada), 40! (id.), 600,602, 603 (id.); Hu 103 (id.), 106 (id.), 109, 112 (id.), 200, 202, 206 (id.), 207 (id.), 302, 304 (id.), 403 (id.), 405, 406, 500, 600, 601, 602 (cucullae); Te 101, 200, 300, 401, 402, 405 .

46. B. Coll y Altabás, Colecciones de voces usadas en la comarca de La Litera, en G. Borao, Diccionario de voces aragonesas, op. cit.

47 Lo $605 ;$ Na 308, 500, 502, 602; Z 302, 303, 500, 501, 505, 506, 507, 602; Hu 406, 407, $408 ; \mathrm{Te} 100,206$

${ }^{48}$ A. $\mathrm{M}^{\mathrm{a}}$ Alcover; F, Moll (colab.); J. Moll, Diccionari català-valencià-balear: inventari lexicogràfic i etimològic de la llengua catalana en totes les seves formes literàries $i$ dialectals, Moll, Palma de Mallorca, 1980-1983. En adelante citaremos por Alcover.

4) El ALEICan en una localidad ( $\mathrm{Lz} 20)$ y el ALEA en dos localidades granadinas y dos de Jaén (Gr 200, 20I; J 400, 402) (Vid. ALEA, II, adición al mapa 408 y ALEICan, I, 308).

51) $S 206,210($ ALEC (ant $, \mathrm{I}, 632)$.

si Hu 108, 112, 201, 205, 400, 401, 402, 403, 404, 601; Te 402, 504.

52 Vid. ALEA, II, adición al mapa 408 y ALEICan, I, 308

5. Lo 400, 604, 605; Z 305; So 400. 
parecido a la golondrina'. García de Diego lo define como 'ave oncejo o vencejo".

20.- Falceta es 'vencejo' en el centro de la provincia de Huesca ${ }^{55}$. Andolz registró la variante falzeta que en ornitología es 'vencejo'.

21.- También el 'vencejo' es ordejo en el centro de Zaragoza ${ }^{56}$.

22.- La forma reviruelo 'avión' se encuentra en un núcleo homogéneo en el centro de La Rioja y en dos puntos de Navarra ${ }^{57}$. Para Iribarren es un 'pájaro más pequeño que la golondrina, de tripa blanca, que suele anidar en las orillas de los ríos'. García de Diego recoge la variante con $b$, rebiruelo como 'avión o vencejo' que es un derivado de ribera.

23.- Picatroncos (y picatronco) designa al pájaro 'picamaderos' de forma general en Aragón, Navarra y Rioja ${ }^{58}$. Iribarren dice que es 'picamaderos, ave del orden de los pájaros'. Andolz y Goicoechea lo definen como 'pájaro carpintero'. Rohlfs señala que es una 'especie de pico, ave'. Andalucía también recogió la voz en varias localidades".

24.- El 'picamaderos' es también denominado repicatroncos en localidades aisladas de Vitoria, Navarra, Zaragoza y Huesca ${ }^{(6)}$. Iribarren afirma que es el 'nombre que dan al pájaro llamado picatroncos'.

25.- Del mismo modo, el vocablo picaguacero representa al 'picamaderos' en tres puntos de Huesca ${ }^{61}$. Para Andolz se trata del 'pico real o picoverde'. Rohlfs lo define como 'picoverde, ave'.

26.- Otra voz, picapino, designa también a 'picamaderos' en algunas localidades de Zaragoza y Huesca ${ }^{62}$. Andolz y González Guzmán ${ }^{6.3}$ aseguran que es cl 'mochuelo'. Aragüés recoge picapín con el sentido de 'pájaro carpintero'. Rohlfs lo marca escuetamente como 'pico, ave'. Y Torreblanca ${ }^{64}$ dice que picapino como 'picamaderos' aparece también en Córdoba y Granada. Sin cmbargo, el ALEA registra picapino en localidades de Jaén y Granada pero no de Córdoba ${ }^{65}$. R. d'Andrés lo define como 'pájaro carpintero'.

5. C. Goicoechea, Vocabulario riojano, Anejo VI del BRAE, Madrid, 1964.

55 Hu 206, 300, 301 (; falcete), 302, 304.

5 Z $\quad$ 501, 502, 503 .

57 Vi 600; Lo 101, 301, 303, 304, 305, 400, 401, 603; Na 306, 309, 403 (riviruelo), 502.

58 Lo 100, 103, 300, 400 (picatronco), 500,601, 602, 603, 604; Na 101 (id.), 102 (id.), 103, $200,201,202,204,205,206,300,301$ (id.), 302 (id.), 303, 305 (id.), 306, 307, 308, 309, 400 (id.), 401 (; id. $), 402,403,404,405,500,501,600,601,602 ; \mathrm{Z} 100,101$ (id.), 200, 201 (id.), 300, 304 (id.), 400, 502; Hu 100, 101, 102, 105 (id.), 107 (id.), 108 (; id.), 110 (id.); Te 203.

59) Al 401, 405, 502,600,602; J 500; Se 501 (ALEA, II, 412).

(1) Vi $600 ; \mathrm{Na} 304 ; \mathrm{Z} 202,305 ; \mathrm{Hu} 303$.

b) Hu $111,203,302$.

(2. Z 200; Hu 103, 104 .

63 P. González Guzmán, El habla viva del Valle de Aragüés, CSIC, Zaragoza, 1953.

s M. Torreblanca, Estudio del habla de Villena y su contarca, Instituto de Estudios Alicantinos, Alicante, 1976.

(5) Gr 200; J 200, 201 (picapinos), 204, 400, 600. 
27.- El 'abejaruco' es picabejero en una localidad de Rioja, en una pequeña zona comprendida cntre el centro y sur de Navarra y en dos pueblos de Zaragoza $^{66}$. Iribarren señala que es 'una clase de pájaro' y Goicoechea afirma que en Rioja llaman así al 'abejaruco'.

28. - La 'oropéndola' recibe el nombre de yeguacero en varias poblaciones navarras, zaragozanas y oscenses ${ }^{67}$. Andolz consideró que era sinónimo de picaverde 'pájaro carpintero' y Rohlfs lo definió del mismo modo.

29.- Gay 'arrendajo' es voz general en Huesca ${ }^{68}$. Andolz, Mott, Borao y Rohlfs lo recogen asimismo como 'arrendajo'.

30.- La 'abubilla' es borbute (y vars. fonéts. burbute, burbut, borbuta, burbuta, borbuz) en varias localidades de Aragón y Navarra ${ }^{69}$. Borbute y borbut 'abubilla' lo documentó Andolz. Rohlfs registró burbute como 'abubilla'.

31.- Gallico de San Martín designa a 'abubilla' en el norte y centro de Navarra, en dos puntos de Zaragoza en contacto y en una localidad de Huesca ${ }^{70}$. Es el 'nombre que dan a la abubilla' en Iribarren. Andolz registró la palabra gallo como equivalente a gallo de San Martín 'abubilla'.

32.- Babuta (y vars. fonéts. bubuta, barbuta) es 'abubilla' en el centro-oeste de Navarra y en cuatro localidades del centro-oeste de Teruel ${ }^{71}$. Iribarren documental babuta y barbuta como 'nombre que dan a la abubilla'. También para Goicoechea es 'abubilla'. García de Diego afirma que babuta es 'la onomatopeya del canto de la abubilla'.

33.- Charlo es 'mirlo' en localidades aisladas de Huesca ${ }^{72}$. Aragüés y Mott lo identifican con el 'zorzal'.

34.- La variante charro designa también al 'mirlo' en un punto de Navarra y cuatro en Huesca' ${ }^{73}$. Iribarren indica que es el 'nombre de cierto pájaro que viene a invernar'. Andolz y Aragüés consideran que es el 'zorzal'. Y Goicoechea señala que es una 'ave de la familia del malviz y de mayor tamaño que él'. Sin embargo, en cl ALEANR csta voz no aparece en ninguna localidad de La Rioja.

(nit) Lo 605; Na 308, 403, 500 (picabejeros), 500, 501, 502, 601; Z 301, 302.

(17 Na 206,403,404; Z 101, 200, 201, 202, 300; Hu 100, 102, 103, 105, 108, 109.

(18) Hu 100,102,103,104,106,107,108,109,110,111,200,201, 202, 203, 204, 205, 206, $207,302,305,402,403,404,405,406,600$.

(a) Lo 600 (bolbuta); Na 403 (burbuta), 404, 600 (borbuta); Z 100, 201, 202, 300, 302 (burbutc), 304 (burlute), 305 (burbui; burbuces), 400, 402 (burbute), 504 (borbuz), 607; Hu 100 (borbut), 101, 102 (burbute), 103, 104 (id.), 105, 107 (; burbut; borbú), 108 (burbute), 109 (bortuta), 110 (burbuta), 112, 300 (; burbute), 301 (id.); Te 100 (borbuz), 101 (id.), 102, 104 (borbuta), 206 (burbut), 300 (burbute), 301 (borburica), 307 (burbut), 308 (barbuta), 402 (bobut), 501 (burbuz), 502 (borbuta).

70 Na 104 (gallo San Martín), 106 (gallico S. M.), 201 (id.), 202 (id.), 203 (gallo S. M.), 206 (gcillico S. M.), 300 (id.), 303 (id.), 305 (id.), 308 (id.), 400 (id.), 40 I (id.), 402, 403, 404; Z 100, 200 (gallo de S. M.), 400 (gallico S. M.); Hu 105.

${ }_{71} \mathrm{Na} 205,304,306,307,309,500,501$ (barbuta); Te 302 (bubuta), 303 (id.), 304 (id.), 308 (barbuta).

$72 \mathrm{Hu} 100,111,203,302$.

$7.3 \mathrm{Na} 302 ; \mathrm{Hu} 110,201,204,403$. 
35.- Chordo es 'tordo' en un grupo de localidades de Zaragoza y Huesca ${ }^{74}$. Para Andolz es el 'tordo de color blanco'. Araguiés lo identifica con el 'tordo' y con el 'mirlo'.

36.- Choa es 'zorzal' en el centro de Navarra ${ }^{75}$. Iribarren define la voz como 'pájaro granívoro parecido al grajo'.

37.- Bobón es 'búho' en tres puntos de Zaragoza y en el norte y centro de Huesca $^{76}$. Etimológicamente $(D C E C H)$, proviene del lat. vg. BUFO. La -Fintervocálica se sonorizó en la forma alto-aragonesa bobón y catalán ribagorzano bobó anotado — según Corominas - en Castrocit. En la segunda acepción de la palabra, el Diccionario aragonés de Andolz registra 'ave rapaz nocturna, búho'. Asimismo, anota la variante bubón como 'búho real'. Benito Coll y Rohlfs identifican la voz como 'búho'.

38.- 'Búho' también es bugo en localidades aisladas de Navarra, Zaragoza y Huesca $^{77}$. Andolz la definió como 'búho'.

39.- La voz latina bufo designa a 'búho' en puntos aislados de Navarra, Zaragoza y Teruel ${ }^{78}$. El $D C E C H$ señala que esta forma se halla en glosas y en manuscritos de San Isidoro y aunque la forma con $-h-$ se halla desde el siglo XIII y es la más común, la forma bufo también aparece en lo antiguo y hoy se emplea en Aragón. Rohlfśs lo define como 'búho'.

40.- El 'búho' también es denominado bubarro en el centro-oeste navarro ${ }^{79}$. Iribarren explica que es una variante de buharro ('ave de rapiña parecida al búho, pero más pequeña').

41.- Chuta es 'lechuza' en Aragón ${ }^{80}$. En los léxicos dialectales de Andolz, Coll y Rohlfs se registra igualmente como 'lechuza'.

\section{Nuevas acepciones}

En el conjunto de voces que designan a las aves silvestres encontramos también un número destacado de ellas que identifican o designan pájaros distintos de los que presentaba el DRAE. Estas voces son:

1.- Culón es 'pajarillo sin pluma' en una localidad riojana, dos en Zaragoza, Huesca y cuatro en Teruel" ${ }^{81}$ El DRAE explica que culón es el que 'tiene muy abultadas las posaderas'. Sin embargo, Iribarren nos aclara que culón es el

$74 \quad$ Z 201, 202; Hu 112.

$75 \mathrm{Na} 303,401,402$.

76 Z 201, 202 (bubón), 400; Hu 103, 106, 107, 108, 109, 110, 111, 112, 204, 205 (bobó), 206, 207, 302, 400, 402, 403, 404 (id.), 405, 406 (id.).

77 Na $501 ; \mathrm{Z} 601 ; \mathrm{Hu} 101,601$.

$78 \quad \mathrm{Na} 304 ; \mathrm{Z} 605 ; \mathrm{Te} 202$ (bufón), 204.

79) $\mathrm{Na} 301,302,306$.

80) Z 606; Hu 101, 402 (chut), 404, 407, 408, 600, 601, 602, 603; Te 202, 205, 207.

8) Lo 305, 501; Z 306, 607; Hu 301, 601; Te 103, 301, 305, 400. 
nombre que dan a la cría de pájaro, cuando está todavía en tripota, en pelo malo, pelo bruja o pelo cañón y que no ha desarrollado hasta alcanzar el pelo bueno. Considera, además, que la cría de pájaro es también denominada hijesno, fillesno y fijerno. La voz la encontramos de nuevo en localidades de Andalucía ${ }^{82}$.

2.- Tarín es 'verderón' en el norte de Navarra y en una localidad burgalesa ${ }^{83}$. El DRAE la localizó apropiadamente como provincialismo navarro ${ }^{84}$ con el significado de 'cierta ave del orden de las paseriformes'. Esta definición resulta insuficiente al no especificar la avecilla a la que se refiere. Iribarren caracteriza mejor a este pajarillo: 'pequeño pájaro de pico cónico y puntiagudo y plumaje cntre amarillo y verdoso, que canta mejor que la cardelina'. Señala, además, que en otras localidades se le conoce como 'verderón' y 'pardillo'.

3.- Cogullada (y cugullada) es 'cogujada' en varias localidades de Aragón ${ }^{85}$. El DRAE deline la voz como 'papada del puerco' que nada tiene que ver con el pájaro 'cogujada'. Corominas en el DCECH también recoge este significado y explica que cogullada es un término catalán que aparece desde principios del siglo XVI, gencralmente en la forma de asimilación vocálica cugullada y afirma que en Benasque se llama cogullada a la 'papada del puerco'. García de Diego la definió igual pero también la señaló como 'cogujada'. Para otros lingüistas, Andolz, Borao, Aragiés y Rohlfs cogullada es 'cogujada'.

4.- La 'cogujada' también recibe el nombre de golloría en una localidad de Rioja, tres de Zaragoza en contacto con una de Teruel y en varias localidades del centro y sur de Teruel, formando una zona compacta ${ }^{86}$. EI DRAE recoge golloría como sinónimo de gollería que significa 'manjar exquisito'. Corominas cree que gulloría 'cogujada' es la palabra engullir por la rareza de la carne de este pájaro como manjar. La acepción antigua de golloría es el nombre de un ave, en griego un pájaro fabuloso 'cogujada'; la cogujada es pájaro que canta al nacer el alba. No asegura Corominas que la cogujada sea efectivamente muy sabrosa, pero sí que se la buscaba a veces y era difícil de obtener. Luego podemos considerar - así lo afirma el etimólogo- que golloría 'cogujada' es un retoño semántico de la acepción fundamental 'golosina, cosa extraordinaria'. Además, asegura que a pesar de la antiguiedad de estas formas, la forma originaria es la moderna y muy usual gollería, ya que es la única que admite una explicación etimológica, pues el paso a golloría se debe a una dilación de la primera vocal, y el influjo de engullir, junto con la acción metalónica de la $i$, explican las demás. Según el P. Sarmiento

82 Vid. ALEA, H, adición al mapa 403.

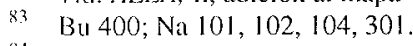

84. Sin embargo, no podemos dejar de observar que en esta ocasión el DRAE localizó una voz que sólo aparecía en cuatro localidades del ALEANR. Hemos visto cómo en otros casos la Academia ignoraba una voz que aparecía en un número mayor de localidades que definían zonas homogéneas.

8.5 Z 402, 605; Hu 108, 201 (cugullada), 205, 400, 401 (id.), 402 (id.), 404, 407; Te 202 (id.), 205 (id.), $400($ id.).

86. Lo 603; Z 502, 505 (golloría moñuda), 507 (id.); Te 101 (gulluría), 307, 308 (id.), 404, 406,502 . 
- dice Corominas- a mediados del siglo XVIII golloria 'cogujada' era propia de Aragón. Si acudimos a los mapas, observamos que la voz está recogida casi exclusivamente en Aragón, porque golloría con el sentido de 'alondra' y 'aguzanieves' lo hemos recogido en Andalucía ${ }^{87}$ y en Rioja ${ }^{88}$. Concluye Corominas que no hay que pensar en derivar gollería del nombre latino de la cogujada, GALERITA, pues sería imposible explicar la pérdida de la -T- en cualquicr lengua romance, salvo el francés, donde no hay huellas de tal palabra latina. Por otra parte, Andolz también registró la voz como 'cogujada'.

5.- Avión es 'vencejo' de forma general en Rioja y en dos localidades zaragozanas". El DRAE lo define únicamente como 'pájaro, especie de vencejo'. Corominas, sin embargo, explica que esta voz proviene probablemente del antiguo gavión (forma que también documentamos en el mapa), de origen incierto, quizá relacionado con el latín GAVIA 'gaviota'. Avión es, seguramente alteración de gavión por influjo de ave. El Diccionario de Autoridades ${ }^{(1)}$ señala que 'en algunas partes y provincias de España lo equivocan con el vencejo y que cn Andalucía truecan los nombres y llaman al 'vencejo' avión y al 'avión' vencejo. Así lo hemos observado en Andalucía donde el término avión es general cn todo el mapa con mayor predominio sobre la voz vencejo ${ }^{21}$. En Cantabria también es general ${ }^{92}$. Si observamos en el mapa del ALEANR correspondiente a 'avión' nos damos cuenta de que el término avión aparece solamente en nueve localidades, frente a las veinticuatro respuestas que encontramos como 'vencejo" $"$.

6.- Aguacero es 'picamaderos' en Huesca"4. La voz consta en el DRAE pero no con el sentido de 'ave'. Andolz lo define como 'pájaro picaverde y lo llaman así porque dicen que anuncia la lluvia'.

7.- El 'abejaruco' recibe el nombre de colmenero en localidades aisladas de Rioja, Aragón y una en Valencia". El DRAE lo define como "persona que tiene colmenas o cuida de ellas'. Por extensión se ha llamado colmenero a este pájaro porque se alimenta de abejas y por lo tanto ataca las colmenas.

87 Gr 511; J 204, 414; Co 104 (ALEA, II, 408).

si Vill nota 83.

sy Bu 400; Vi 300, 600; Lo 100, 101, 102, 103, 300, 301, 302, 303, 304, 305, 401, 500, 501, 502,$601 ;$ Na 500 (avrisin); Z 500, 506; So 600; Gu 200, 400.

(y) Real Academia Española, Diccionario de Autoridades, cdición facsímil, Gredos, Madrid, 1979, 3 tomos.

1) Vid. $A L E A, 11,410$.

y? Vid. ALEC Cint, $\mathrm{I}$, adición al mapa 631.

Cf. ALEANR, IV 455.

Hu 206, 207, 301, 305, 400, 403, 405, 500 (aguaceros).

15 Lo 304,$602 ; \mathrm{Z} 505 ; \mathrm{Hu} 108$; Te 304; V 101. 
8.- Abejorro es 'abejaruco' en algunas localidades de Huesca y una de Navarra". El Diccionario académico lo define simplemente como 'insecto'. Sin cmbargo, Andolz y Rohlfs documentan la acepción 'abejaruco'.

9.- Lal voz engañapastor identifica a la 'aguzanieves' en la franja oriental del ALEANR, incluida Navarra' ${ }^{97}$. El DRAE registra la voz con el significado de 'chotacabras'. El pájaro chotacabras recibe el nombre de 'engañapastor' en un número importante de localidades del $A L E A N R^{98}$. Sin embargo, con el significado de 'aguzanieves' lo encontramos en Andolz, Araguiés, Torreblanca y Rohlfs. Alcover explicó que está compuesto etimológicamente por el imperativo de engañar y por cl sustantivo plural pastores, y considera que esta denominación se puede explicar por la opinión muy extendida que atribuye a este pájaro la costumbre de ir por las noches a chupar la leche de las cabras y ovejas (de aquí le viene cl nombre de chotacabras), y también porque este pájaro parece tener poco miedo de los campesinos, ya que aunque se le acerquen, él sigue caminando o está detenido cn el suelo y parece que se va a dejar capturar, pero cuando van a agarrarlo, toma el vuelo y se va. De forma parecida González Ollé explicó que cra cl "pájaro caracterizado por no levantar el vuelo hasta el momento en que parece que va a ser cogido'. A su vez, Iribarren señala que es el 'nombre que dan al pájaro llamado motacilla alba, al que llama también pájaro o pajarita de las nieves. Es un pajarillo gris, con el pecho blanco, que puede andar en invierno en Ios labrados'.

10.- Nevero es también 'aguzanieves' en localidades de Navarra, una en Zaragoza, otra en Huesca y cuatro en Teruel ${ }^{99}$. La voz figura en el DRAE, pero no con el sentido de 'ave'. Iribarren dice que es el 'nombre que dan a la pajarita de las nieves o motacilla alba'. Goicoechea recoge en su Vocabulario la definición que Echavarría da para la voz: 'en Rioja llaman así a un pajarito del tamaño de un gorrión, cl cual no se ve en los poblados sino en tiempos de nieves, por lo cual le dicron este nombre. Es muy hermoso por la variedad de colores que tiene, unos reales y otros cambiantes o aparentes; su cabeza está coronada con un copetito gracioso que oculta y maniliesta en ocasiones. Su habitación es en los vergeles, donde cría, y su canto es muy subido, aunque no muy agradable. No es mosquero, y se alimenta por lo común de semillas'. Curiosamente, esta voz no se registró en

96 Na 203; Hu 103, 300,600.

97 Na 106, 201, 206 (engañapastó), 300, 301, 304, 306, 308, 405, 500; Z 200, 303, 402, 601, 603, 604, 605, 606 (id.); Hu 100 (; engañapastores), 104, 106, 109, 110, 111, 112, 200, 201 (engañapastó), 202 (engañapastós), 204 (id.), 206, 207, 301, 302, 303, 305, 403, 600, 601, 602, 603; Te 203, 204 (id.), 205, 207 (id.), 401, 600; Cs 302.

is Bu 400; Vi 300, 600; Lo 100, 300, 301, 302 (engañapastores), 401, 500; Na 205, 302, 303, $304,305,403,405$ (id.), 600 (id.); Z 101 (id.), 200, 201, 202 (id.), 400, 402, 600; Hu 103 (id.), 105 (id.). 107 (id.) 108, 300, 304, 405, 407, 500; Te 307 (id.), 403, 405 (id.), 504; V 100 (ALEANR, IV, 461 ).

(y) Na 306, 309 (neverillo), 600; Z 504; Hu 108; Te 100 (pájaro nevero), 103, 203, 303; Cil 200 . 
ninguna localidad riojana del ALEANR. Eugenio Miguélez ${ }^{1(1)}$ registró nevera como 'pájaro aguzanieves'.

11.- El 'tordo' es tordancha en Navarra ${ }^{101}$. El Diccionario académico incluye en su corpus tordancha como 'estornino', voz, además, típica de Navarra. Sin embargo, en el ALEANR no documentamos en ningún punto tordancha como 'estornino'. En cambio, con el significado de 'tordo' lo volvemos a encontrar en tres localidades de Almería ${ }^{102}$. Iribarren cree que es el nombre que dan al estornino. Para Corominas tordancha, derivado de tordo tiene todo el aire de ser un mozarabismo $(D C E C H)$.

12.- Cabrero es 'chotacabras' en el norte de Huesca ${ }^{103}$. En el DRAE aparece la voz como 'pájaro poco más grande que el canario, de cabeza negra con listas blancas y cuerpo amarillo anaranjado con una mancha verdosa en el lomo. Abunda en la isla de Cuba, donde anida hasta en la jaula'. Poco parece tener que ver esta ave con el chotacabras ${ }^{104}$.

13.- Campero 'gorrión campestre' lo encontramos exclusivamente en Rioja y Navarra ${ }^{11.5}$. EI DRAE lo define como 'perteneciente o relativo al campo'.

\section{Otras localizaciones}

En el análisis que hemos realizado de los nombres de las «aves silvestres» en el ALEANR hemos observado también que el DRAE, en ocasiones, no tenía en cuenta la localización exacta que de las voces nos ofrecen los atlas lingüísticos y que dan testimonio claro de la vitalidad de las denominaciones. Veamos algunos casos:

1.- Pardal es 'gorrión campestre' en localidades de Aragón ${ }^{106}$. En la cuarta acepción del DRAE encontramos la voz con el sentido de 'gorrión'. Corominas señala que este vocablo se halla en castellano «en franca decadencia», ya que perdió terreno a favor de la voz gorrión debido a las connotaciones semánticas

I(*) E. Miguélez Rodríguez, Diccionario de las hablas leonesas: León-Salananca-Zamora, [E] Autor], León, 1993.

101 $\mathrm{Na} 100,300,303,401$ (tordincha).

Ii2 Al 403, 405, 600 (ALEA, II, 418).

103 Hu 100 (crubero), 109, 111 (id.), 200 (craberet), 202 (craber), 312 (crabero).

104 Según el Diccionario académico el chotacabras es el 'ave insectívora, de unos 25 centímetros de largo, pico pequeño, fino y algo corvo en la punta, plumaje gris con manchas y rayas negras en la cabeza, cuello y espalda, y algo rojizo por el vientre, collar incompleto blanquecino, varias cerdillas alrededor de la boca, ojos grandes, alas largas y cola cuadrada. Es crepuscular y gusta mucho de los insectos que se crían en los rediles, adonde acude en su busca, por lo cual se ha supuesto que mamaba de las cabras y de las ovejas'. Hay que decir, además, que a pesar de la extensa y completa definición que la Academia da de este pájaro la voz chotacabras se recogió en una localidad de La Rioja (Lo 103) y no se registró en ningún otro atlas lingüístico. Como vemos, el $D R A E$ no tiene en cuenta la localización geográfica que para cada voz ofrecen los mapas lingüísticos a la hora de marear una voz como general o dialectal.

101 Lo $101,102,103,303,400 ;$ Na 306, 307, 309.

เ166 Z 100, 101, 201, 301, 402; Hu 100, 102, 602; Te 204, 205. 
que empezaba a adquirir el término pardal ${ }^{107}$. Asegura el etimólogo que la voz pardal, se emplea todavía en el aragonés de Graus ${ }^{108}$, en Murcia y en Asturias, y con sentidos secundarios en Salamanca y otros lugares.

2.- Verderol es 'verderón' de forma general en Aragón y en algunas localidades de Navarra en estrecho contacto con Zaragoza ${ }^{109}$. El DRAE lo identifica con el 'verderón' sin connotación de regionalismo. El resto de atlas linguiísticos estudiados nos permite comprobar que la voz no es recogida en Andalucía, Canarias y Cantabria. Por otra parte, Corominas argumenta que la voz es propia del valenciano, aunque como vemos también lo es del aragonés.

3.- Gayo es 'arrendajo' en varias localidades de Rioja, dos grupos en Navarra: uno al noroeste y otro al centro-oeste, tres localidades en Zaragoza y uno en Huesca y varias en Teruel ${ }^{110}$. No aparece esta voz en el ALEA, ALEICan y ALECant. En opinión de Corominas, este término persiste todavía en Álava, Navarra y Aragón. Con estos datos sería lógico pensar que la Academia incluiría la voz en su Diccionario teniendo en cuenta la distribución geográfica que apuntaba Corominas. Sin embargo, la voz aparece como general en el DRAE a pesar de que, según afirma Iribarren, esta voz era incluida por el $D R A E$ como usada en Álava, Aragón y Navarra.

4.- Tordancha es 'tordo' en Navarra'l'. El Diccionario académico lo define como 'estornino' y señala que es voz típica de Navarra. Según el ALEANR, con este significado no es usada en Navarra.

5.- El 'estornino' también recibe la denominación de tordo en algunas localidades navarras ${ }^{112}$. En la tercera acepción del DRAE encontramos tordo como 'estornino', aunque el Diccionario señala que es voz utilizada en América Central, Argentina y Chile.

\section{Conclusiones}

En las páginas precedentes hemos podido comprobar cómo una pequeña parcela de nuestro léxico (el de las 'aves silvestres') en una zona determinada

107 La acepción más extendida de este vocablo es 'miembro viril',

168 Como podemos observar en la nota 105 , el número de localidades aragonesas en las que aparece la voz es mayor.

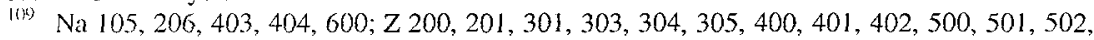
$504,507,600,601,602,604,605 ; \mathrm{Hu} 100,101,102,103,104,105,106$ (verderal), 107, 108, 109, $110,112,200,201,202$ (verderoy), 204, 205, 206, 207, 300, 301, 302, 303, 304, 305, 400, 401, 402 (verdersla), 403, 404, 405, 406, 407, 408, 500,600,601, 602, 603; Te 100, 101, 102, 104, 200201 , $202,203,204,205,206,207,300,301,303,304,305,306,308,400,402,403,404,406,500,501$, $502,503,504,600,601$; Cu 200, 400; V 100; Cs 300, 301, 302.

110 Bu 400; Vi 300, 600; Lo 102, 300, 304, 605; Na 101, 102, 104, 106, 300, 301, 302, 303, 304,

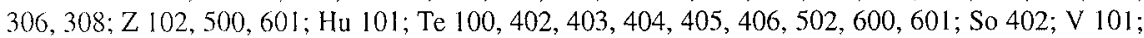
Cs $300,301,302$.

I1! Na 100, 300, 303, 401 (tordincha).

112 Na 101, 102, 104, 206. 
(aquella que nos facilita el $A L E A N R$ ) presenta gran cantidad de casos que atestiguan una vez más el olvido al que, por parte de la Lexicografía, están sometidos los materiales que la Geografía Lingüística pone al alcance de esta disciplina. Hemos visto cómo voces que tenían una vigencia más o menos general en una provincia han aparecido como voces generales, o no lo han hecho; y aquéllas que el diccionario académico marcaba como regionalismos, en el mapa aparecían en un número muy pequeño de localidades y, en cambio, olvidaba marcar otras que presentaban un uso muy generalizado en una determinada provincia. Todo esto nos lleva a probar, una vez más, que la Academia no tiene ni utiliza un criterio determinado para incluir en su diccionario las voces dialectales que por su uso y localización geográfica son utilizadas en la lengua hablada. La importancia de indicar en el diccionario general de la lengua el ámbito exacto de difusión geográfica de una voz cuando ésta no es el término oficial no es una cuestión trivial. Así lo aseguraba Gregorio Salvador cuando manifestaba que

las cucstiones geográfico-lingüísticas no son secundarias, ni mucho menos, en la consideración global del léxico de una lengua, y que el Diccionario general debe ofrecer, con preferencia a los nombres de cosas muy localizadas, los nombres bien localizados de las cosas conocidas ${ }^{113}$.

Sin embargo, no es fácil realizar esta tarea sin atlas lingüísticos que cubran todo el dominio lingǘstico, pero sigue diciendo G. Salvador que

Ya van siendo bastantes los [atlas] que hay y los lexicógrafos tienen la obligación de utilizarlos hasta el máximo de sus posibilidades, no se pueden permitir el lujo de desdeñar la información que los atlas lingüísticos proporcionan $^{1 / 4}$.

Hemos querido presentar una pequeña parte del inmenso material que nos ofrece en este caso el $A L E A N R$ para mostrar una vez más que la Geografía Lingüística ofrece a otras disciplinas lingüísticas una gran cantidad de datos que son neccsarios para inventariar, clasificar y analizar el léxico de una lengua. El estudio de los atlas lingǘrticos españoles contribuye a que nuestra lengua, reflejo de nuestra cultura, se vea cada vez más enriquecida con todas las aportaciones que la Geografía Linguiística pone al alcance de nuestra mano. Con este trabajo pretendemos aportar un granito de arena más a todos los que han denunciado esta realidad: la Lexicografía, en adelante, no debe dar un paso más sin la estimable ayuda de la Geografía Lingüística.

11.3 G. Salvador, "Voces aragonesas en el DRAE», en Actas del I Congreso de Lingüistas Arcigoneses, Dcpartamento de Cultura y Educación, D.L., Zaragoza, 1991, págs. 21-27: pág. 23.

11.4 Lor. cit., pág. 23. 


\section{Índice de términos citados}

abejorro, II. $\$ 8$.

aguacero, II, \$6.

aguaccros, nota 94 del capítulo II.

aloda, I. \$ 14.

aloea, nota 42 del capítulo $\mathrm{I}$.

aloya, I. $\$ 14$.

aloya real, nota 42 del capílulo I.

ave, II. \$5.

avión, II. $\$ 5$.

avrión, nota 89 del capítulo II.

babula, I. \$32.

barbuta, I. \$ 32; nota 69 del capítulo I;

nota 71 del capítulo 1.

bobó, I. \$ 37; nota 76 del capítulo I.

bobón, I. \$ 37 .

bobut, nota 69 del capítulo I.

bolbuta, nota 69 del capítulo 1 .

borbú, nota 69 del capítulo I.

borburica, nota 69 del capítulo I.

borbut, I. \$30; nota 69 del capítulo I.

borbuta, I. $\$ 30$; nota 69 del capítulo I.

borbutc, 1 \$ 30 .

borbuz, I. \$30; nota 69 del capítulo I.

bubarro, 1. $\$ 40$.

bubón, I. \$37; nota 76 del capílulo I.

bubuta, I. \$32; nota 71 del capítulo I.

bulo, 1. \$39.

bufón, nota 78 del capítulo I.

bugo, I. \$38.

buharro, 1. \$4 40 .

burbú, nota 69 del capítulo I.

burbuces. nota 69 del capílulo I.

burbut, I. \$ 30; nota 69 del capítulo I.

burbuta, I. \$ 30; nota 69 del capítulo I.

burbute, I. \$30; nota 69 del capítulo I.

burbuz, nota 69 del capítulo I.

cabrero, II. \$12.

callandra, I. $\$ 18$.

caliandra, I. \$ 17.

campero, II. $\$ 13$.

coculo, I. \$9; nota 25 del capítulo I.

cocullada, I. \$ 16; nota 45 del

capítulo I.

cogullada, II. \$3. colmenero, II. $\$ 7$.

craber, nota 103 del capítulo II. craberet, nota 103 del capítulo II. crabero, nota 103 del capítulo 1. coculo, I. \$ 9 .

cubulo, nota 25 del capítulo I.

cuclillo, I. \$ 10 .

cuco, I. \$ 10.

cucullada, I. $\$ 16$.

cucullae, nota 45 del capítulo I.

cucullo, I \& 11 .

cugulo, nota 25 del capítulo 1 .

cugullada, II. \$ 3; nota 85 del

capítulo II.

culón, II. \$1.

cuquillo, I. \$11.

charlo, I. \$ 33 .

charro, I. \$ 34 .

chilanda, nota 19 del capítulo I.

chilandra, nota 19 del capítulo I.

chilandre, I. $\$ 5$.

chileandrea, nota 19 del capítulo I.

chilandro, nota 19 del capítulo I.

choa, I. $\$ 36$.

chordo, I. $\$ 35$.

chotacabras, II. \& 9.

chut, nota 80 del capítulo I.

chuta, I. $\$ 41$.

engañapastó, nota 97 del capítulo II.

engañapastor, II. \$ 9.

engañapastores, nota 97 del capítulo II;

nota 98 del capítulo II.

engañapastós, nota 97 del capítulo II.

engañar, II. \$ 9 .

engullir, II. $\$ 4$.

en pelo bruja, I. $\$ 2$.

en pelo bueno, I. $\$ 2$.

en pelo güeno, nota 15 del capítulo 1 .

en pelo malo, II. \& 1 .

en tripota, II. $\S 1$.

falceta, I. \$20.

falcete, nota 55 del capítulo I.

falzeta, I. \$20.

fijerno, II. $\$ 1$. 
fillesno, I. $\$ 1 ;$ II. $\$ 1$.

gallico de San Martín, I. § 31 .

gallico San Martín, nota 70 del

capítulo I.

gallo, I. $\$ 31$.

gallo de San Martín, I. § 31; nota 70

del capítulo I.

gallo San Martín, nota 70 del

capítulo I.

gavión, II. $\$ 5$.

gay, I. $\$ 29$.

gayo, III. $\$ 3$.

gollería, II. $\$ 4$.

golloría, II. \$ 4 .

golloría moñuda, nota 86 del capítulo II.

gorrión, III. § 1 .

gulloría, II. $\$ 4$.

gulluría, nota 86 del capítulo II.

hijesno, II. $\$ 1$.

loda, nota 40 del capítulo I.

loea, nota 42 del capítulo 1.

motacilla alba, II. § 9; II. § 10.

nevera, II. \$ 10 .

neverillo, nota 99 del capítulo II.

nevero, II. \$ 10 .

ocete, l. $\$ 19$.

ordejo, I. $\$ 21$.

pájaro nevero, nota 99 del capítulo II.

paparroy, nota 24 del capítulo I.

papirrey, nota 24 del capítulo I.

papirrojo, I. § 8; nota 24 del capítulo I.

papirroy, I. \$ 8; nota 24 del capítulo I.

papitroyo, I. $\$ 8$.

paporroy, nota 24 del capítulo I.

pardal, III. \$1.

pastores, II. \$9

pecu, I. $\$ 10$.

pelo bruja, I. \$2; II. \$1.

pelo bueno, II. $\$ 1$.

pelo cañón, II. § 1 .

pepirroy, nota 24 del capítulo I.

picabejcro, I. \$27.

picabejeros, nota 66 del capítulo I.

picaguacero, I. $\$ 25$.

picapín, I. $\$ 26$.

picapino, I. $\$ 26$. picapinos, nota 65 del capítulo I.

picatronco, I. $\$ 23$; nota 58 del capítulo I.

picatroncos, I. $\$ 23$.

picaverde, I. $\$ 28$.

pinchán, I. $\S 6$.

pinzán, I. $\$ 7$.

piparrayo, nota 24 del capítulo I.

piparroyo, I. § 8; nota 24 del capítulo I. pipirroyo, I. § 8; nota 24 del capítulo I.

recachadera, I. $\$ 15$.

reiseñor, I. \& 12.

rebiruelo, I. $\$ 22$.

repicatroncos, I. $\$ 24$.

reviruelo, I. $\$ 22$.

ribera, I. $\$ 22$.

riviruelo, nota 57 del capítulo $\mathrm{I}$.

tarín, II. $\$ 2$.

tordancha, II. \$11; III. \$4.

tordincha, nota 101 del capítulo II;

nota 111 del capítulo III.

tordo, II. § 11; III. § 5.

vencejo, II, $\$ 5$.

verdelón, I. \$ 12.

verderal, nota 109 del capítulo III.

verderol, III. § 2.

verderola, nota 109 del capítulo III.

verderoy, nota 109 del capítulo III.

voladero, I. $\$ 4$.

volandero, I. § 4.

yeguacero, I. $\$ 28$. 


\section{Referencias bibliográficas}

ALCOVER, ANTONI Ma; F. MOLL (colab.); J. MOLL, Diccionari catalàvalencià-balear: inventari lexicogràfic i etimològic de la llengua catalana en totes les seves formes literàries $i$ dialectals, Moll, Palma de Mallorca, 19801983.

ALONSO GARROTE, SANTIAGO, El dialecto vulgar leonés hablado en Maragatería y Tierra de Astorga, Instituto Antonio de Nebrija, CSIC, Madrid, 1947 (2a edición).

ALVAR, MANUEL, con la colaboración de A. Llorente, G. Salvador y J. Mondéjar, Atlas Lingüístico y Etnográfico de Andalucía (6 tomos), Universidad de Granada-CSIC, Granada, 1961-1973.

—_- con la colaboración de A. Llorente, T. Buesa y E. Alvar, Atlas Lingüístico y Etnográfico de Aragón, Navarra y Rioja, Departamento de Geografía Lingüística, Instituto Fernando el Católico de la Excma. Diputación de Zaragoza, CSIC, Zaragoza, 1979-1983, 12 tomos.

- Atlas Lingüístico y Etnográfico de Cantabria, Ed. Arco Libros, Madrid, 1995, 2 tomos.

- Atlas Lingiilístico y Etnográfico de las Islas Canarias, Ediciones del Excmo. Cabildo Insular, Las Palmas, tomo I, 1975; tomo II, 1976; tomo III, 1978.

— «Proyecto de un atlas lingüístico de Aragón», recopilado en Estudios de Geografía Lingiilistica, Paraninfo, Madrid, 1990.

ANDOLZ, RAFAEL, Diccionario Aragonés, Mira, Zaragoza, 1992 (4ª edición). ANDRÉS, R. D', Diccionario Temáticu Asturianu, Alborá Llibros, Asturias, 1991.

ARAGÜÉS, CHUSÉ, Dizionario Aragonés-Castellán. Castellano-aragonés, Ligallo de Fablans de l'aragonés, Zaragoza, 1989.

BORAO, GERÓNIMO, Diccionario de voces aragonesas, Diputación Provincial de Zaragoza, Zaragoza, 1908.

BUESA OLIVER, TOMÁS, Estudios filológicos aragoneses, Universidad, Zaragoza, 1989.

COLL Y ALTABÁS, BENITO, Colecciones de voces usadas en la comarca de La Litera, en G. Borao, Diccionario de voces aragonesas.

COROMINAS, JUAN Y J. A. PASCUAL, Diccionario Crítico Etimológico Castellano e hispánico, Gredos, Madrid, 1980-1991, 6 tomos.

DÍEZ, Ma SOLEDAD, Léxico leonés, Universidad de León, León, 1994.

GARCÍA DE DIEGO, VICENTE, Diccionario etimológico español e hispánico, Saeta, Madrid, 1969 (2 $2^{a}$ edición).

GOICOECHEA, CESÁREO, Vocabulario riojano, Anejo VI del BRAE, Madrid, 1964. 
GONZÁLEZ GUZMÁN, PASCUAL, El habla viva del Valle de Aragüés, CSIC, Zaragoza, 1953.

GONZÁLEZ OLLÉ, FERNANDO, El habla de la Bureba. Introducción al castellano actual de Burgos, anejo LXXXVIII de la RFE, CSIC, Madrid, 1964.

IRIBARREN, JOSÉ Ma , Vocabulario navarro, Ed. Gómez, Pamplona, 1952.

MIGUÉLEZ RODRÍGUEZ, EUGENIO, Diccionario de las hablas leonesas:

León-Salamanca-Zamora, [El Autor], León, 1993.

MOTT, BRIAN, Diccionario Chistavino-Castellano, Caja de Ahorros de Aragón y Rioja, 1978.

NAVARRO CARRASCO, ANA ISABEL, El Atlas de Canarias y el Diccionario Acaclémico, Universidad, Alicante, 1996.

REAL ACADEMIA ESPAÑOLA, Diccionario de Autoridades, edición facsímil, Gredos, Madrid, 1979, 3 tomos.

_- Diccionario de la Real Academia Española, Espasa-Calpe, Madrid, 1992, $21^{\text {* edición. }}$

ROHLFS, GERHARD, Diccionario dialectal del Pirineo Aragonés, Institución Fernando el Católico, Zaragoza, 1985.

SALVADOR, GREGORIO, «Voces aragonesas en el DRAE», en Actas del I Congreso de Linguiistas Aragoneses, Departamento de Cultura y Educación, D.L., Zaragora, 1991.

TORREBLANCA, MANUEL, Estudio del habla de Villena y de su comarca, Instituto de Estudios Alicantinos, Alicante, 1976. 